\title{
7. Norddeutsche Fragmente mit Lautenmusik um 1460 in Wolfenbüttel
}

1. Das im Folgenden zu behandelnde Dokument hat sich im Manuskript VII B Hs 264 des Staatsarchivs Wolfenbüttel gefunden. ${ }^{1}$ Dieses Manuskript besteht aus 19, von einem mit braunem Leder halb überzogenen Holzdeckelband, heute ohne die alte Schließe, zusammengehaltenen, paginierten Pergamentblättern von 22,6 cm (Höhe) x 16,2 cm und enthält in seinem Hauptcorpus die Statuta Ecclesiae ad Montem Sancti Cyriaci 1483, wie sie auf p. $1\left(=\right.$ fol. $<1>$ ) eine Hand des 17. Jahrhunderts betitelt hat. ${ }^{2}$ Auf p. 2 (fol. $<1^{\prime}>$ ) steht ganz am unteren Rand ein - von der Schrift her älteres - Abgabenverzeichnis, ${ }^{3}$ und auf p. 3-34 der Text der im Titel angekündigten Statuten dieses auf einer kleinen Anhöhe außerhalb des alten Braunschweig gelegenen Kollegiatsstiftes; ${ }^{4}$ angefügt sind Ergänzungen aus dem Jahre 1536. Die Seiten 35-38 sind vorliniert, aber nicht beschrieben. Sowohl Abgabenverzeichnis als auch Statuten nennen Personennamen, das erste diejenigen von zum Teil sogar noch vor dem 15. Jahrhundert abgabenpflichtigen Persönlichkeiten außerhalb des Stiftes, die zweiten die Namen jener Kanoniker des Stiftes St. Cyriacus selbst, welche diese Statuten im April 1483 verabschiedet haben. Ihre vorliegende Niederschrift in der, wie ausdrücklich vermerkt, von Rom gebilligten Form ist nicht vor Anfang 1485, dann aber, angesichts ihres wichtigen kirchenrechtlichen Charakters, wohl sehr bald vollzogen und gebunden worden; die beiden Einzelstempel

1 Für den freundlichen Hinweis auf die Fragmente sei Herrn Dr. Helmar Härtel (Wolfenbüttel) gedankt, der bei der Durchsicht der Handschriftenbände des Niedersächsischen Staatarchivs Wolfenbüttel auf sie stieß. Dank gilt auch den Herren Kollegen Jürgen Heidrich (Münster/W.) und Dr. Joachim Lüdtke (Fürth) für lautentechnische und -historische Auskünfte; Jürgen Heidrich hat auch das Manuskript dieses Abschnitts gelesen und förderliche Ratschläge gewährt.

2 Eine Beschreibung des Gesamtmanuskripts gibt Jörg Schillinger, Die Statuten der Braunschweiger Kollegiatsstifte St. Blasius und St. Cyriacus im späten Mittelalter, Hannover 1994 (= Quellen und Studien zur Geschichte des Bistums Hildesheim, Bd. 1), S. 32-40.

3 Abdruck bei Schillinger (s. oben, Anm. 2), S. 88, zu fol. 1'.

4 Zur Geschichte des Stifts St. Cyriacus überhaupt vgl. Ernst Döll, Die Kollegiatsstifte St. Blasius und St. Cyricaus zu Braunschweig, Braunschweig 1967 (= Braunschweiger Werkstücke, Bd. 36), passim. 
auf dem Ledereinband - fünfdoppelblättrige umrandete Rosette bzw. kleine Drei- und Fünfkugel-Ornamente in Rhombus, das Ganze in großräumigem rechteckigem und diagonalem Doppellinien-Gerüst - gehören einer nicht namentlich bekannten Braunschweiger Werkstatt, ${ }^{5}$ die mit zwei weiteren teilweise übereinstimmenden Stempelbelegen auf Einbänden von Drucken von 1483 und 1493 in Braunschweig und Wolfenbüttel bezeugt ist, ${ }^{6}$ aber zu einer genaueren Datierung nichts Weiterführendes beibringen kann; der vorliegende Einband dürfte, dem Inhalt entsprechend, aus dem Jahre 1485 stammen.

Die Innendeckel des Bandes sind vorn und hinten mit Papierspiegeln abgedeckt worden, die heute von den Deckelflächen abgelöst, aber im Band eingebunden geblieben sind (vgl. Abb. 7a)-d)). Beide Blätter, ohne Foliierung oder Paginierung, sind auf allen ihren vier Seiten mit Noten beschrieben: vom Buchbinder bei fol. A am inneren, bei fol. A und B auch je am oberen Blattrand, aber ohne Textverlust beschnitten, messen die beiden Folien vorn ca. $22 \mathrm{~cm}$ (Höhe) x ca. 15,8 cm, hinten ca. $22 \mathrm{~cm}$ (Höhe) $\mathrm{x}$ ca. 15,2 cm; die ehemals auf die Innendeckel aufgeklebten Seiten, also fol. A und B', sind durch den Kleister etwas gebräunt, fol. A unten rechts auch beschädigt. Das dafür verwendete Papier trägt kein Wasserzeichen; nach dem Schriftcharakter der erscheinenden Textmarken und dem Repertoire der eingetragenen Sätze - das, soweit musikalische Konkordanzen bzw. Vergleichsüberlieferungen vorliegen, deutlich in die Zeit um 1450-65 weist - dürfte die Niederschrift um 1460 erfolgt sein. Diese Datierung wird durch die Überlegung bekräftigt, daß der Buchbinder des Gesamtbandes im Jahre 1485 gewiß nicht inhaltlich aktuelles, vielmehr bereits unzweifelhaft veraltetes Fragmentenmaterial für die Abdeckung der Innenseiten der Deckel wiederverwendet habe. Der oben erwähnte Wolfenbütteler Parallelband aus der gleichen Bindewerkstatt zeigt ebenfalls Papierspiegel, und zwar solche, die in darauf aufgezeichneten Wirtschaftsbetrieb-Listen auf wohl braunschweigische und deutlich klösterliche Umgebung hinweisen

5 In Teilen entspricht die Prägung derjenigen der Einbände der namentlich nicht bekannten Braunschweiger „Kleinfiguren-Meister“-Werkstatt; freundlicher Hinweis von Herrn Dr. Helmar Härtel, Wolfenbüttel. Zu dieser Werkstatt vgl. Ilse Schunke/Konrad von Rabenau, Die Schwenke-Sammlung gotischer Stempel- und Einbanddurchreibungen, Bd. II, Berlin 1979, bes. S. 45f.; wenigstens der Rosetten-Stempel findet sich abgebildet bei Ilse Schunke, ebda., Bd. I, S. 269, Nr. 552.

6 Aurelius Augustinus, Liber epistolarum, Basileae, Johann Amerbach, 1493 (Stadtbibliothek Braunschweig), und Jordanus de Quedlinburg, Opus postillarum et sermonum de euangelijs dominicalibus, Argentine 1483 (Herzog August Bibliothek Wolfenbüttel). Frau Gundula Schmidt, Stadtbibliothek Braunschweig, sei auch hier für freundliche Auskünfte bestens gedankt. 
und wiederholt das Datum schon von 1439 bieten. Das könnte bedeuten, daß die Niederschrift der Fragmente im Stift St. Cyriacus selber erfolgt ist. Sicher ist dies allerdings nicht, da eine eigene Klosterbuchbinderei in St. Cyriacus nicht nachgewiesen ist; auch eine Identifikation des auf fol B' notierten Namens ,Joh:<annes>,, (einzige Eintragung nicht in dunkelbraun-schwärzlicher, sondern roter Farbe) mit den zu Beginn der oben erwähnten Statuten aus St. Cyriacus genannten Kanonikern Johannes Schorkop oder Johannes Mysner ${ }^{7}$ bleibt spekulativ, wenngleich denkbar; aber auf jeden Fall darf man vermuten, daß die Fragmente in oder in der näheren Umgebung von Braunschweig beschrieben worden sind. - Auf fol. $A^{\prime}$ und $B$ finden sich schließlich am unteren bzw. am äußeren und unteren Rand und im Blattinneren Federproben, vielleicht von dritter Hand, darunter auch Notenproben; eine Vermutung, es könnten die Zahlen „39“ (fol. A') und „22“ (?, fol. B) auf eine Numerierung der Stücke hinweisen, ist wegen der Stellung dieser Zahlen auf den beiden Seiten sehr zweifelhaft.

2. Die folgende Übersicht über den Inhalt der beiden Notenblätter reiht diese so, wie sie im Trägerband verarbeitet worden sind. Um nicht den Eindruck zu erwecken, sie hätten auch in ihrer musikalischen Provenienzhandschrift in der gleichen Folge gestanden, werden sie nicht numeriert, sondern mit Buchstaben gekennzeichnet; eine direkte inhaltliche Verbindung von fol. A und B oder umgekehrt durch ihre Eintragungen ist nicht gegeben. Trotzdem läßt die Niederschrift der auf beiden Blättern lückenlos hintereinander notierten Sätze vermuten, daß die Provenienzquelle einen gewissen Umfang besaß; die Beschneidung der Blätter an ihrem oberen Rand und einmal auch ihrer linken Seitenkante hat wohl eine alte Foliierung beseitigt, wenn es eine solche denn einmal gegeben hat. Auf die Problematik der „Konkordanzen“ und deren Benennung wird an späterer Stelle eingegangen. ${ }^{8}$

A: Systeme 1-2: <Cum lacrimis $>, 2$. pars, Schluß

Systeme 2-4: $3^{a}$ pars Cum lacrimis, Anfang $\}$ anon. [Johannes Ciconia?]

A': Systeme 1-2: <Cum lacrimis $>$, 3. pars, Schluß

$\mathrm{K}:-$ ?

N.B.: Keine sichere Übereinstimmung mit dem Con lagreme-Satz 2v. von Ciconia oder dem anonymen Con lagreme-Satz in Paris 6771, fol. 27'.

7 Vgl. Schillinger (s. oben, Anm. 2), S. 88, zu fol. 2.

8 Vgl. unten, S. 78f. 
Systeme 3-4: Myn trud gheselle, nur erste Hälfte; anon.

K: - Berlin 40613, p. 38-39; 3v., anon.

- München 810, fol. 9' (Nr. 21) ; 3v., anon.

N.B.: Verarbeitet ist der D des K-Satzes in Berlin 40613 und München 3725.

B: Systeme 1-4: Gruß senen jch im hertzen traghe; anon.

K: - München 810, fol. 57'-58 (Nr. 49); 3v., anon.

N.B.: Verarbeitet ist der D des K-Satzes in München 810 .

B': Systeme 1-2: Jch fare do hyn wen eß muß syn; anon.

K: - Berlin 40613, p. 9; nur 1v., anon.

N.B.: Verarbeitet ist, und zwar mehrfach in der Mittellage, die K-Liedmelodie in Berlin 40613.

Systeme 3-4: Elende du hest vmb vanghen mich: anon.

K: - Berlin 40613, p. 5; nur 1v., anon.

N.B.: Verarbeitet ist, und zwar in der Mittellage, die K-Liedmelodie in Berlin 40613.

3. Die verwendete Musiknotation wird den Leser staunen lassen, denn er hat eine solche mit Sicherheit so noch nie gesehen. Gewiß, die vier erhaltenen Seiten lassen keinen Zweifel daran, daß Musik aufgezeichnet ist: es zeigen sich achtlinige, auffallend zuverläßig von $\mathrm{g}$ bis $\mathrm{g}^{\prime \prime}$ geschlüsselte Systeme und mit „Köpfen“, Cauden und Fahnen versehene, demnach fraglos mensural zu denkende Musiknoten. Aber es muß sich sofort die Frage nach Wesen und Verwendungsabsicht der hier vorliegenden Notation stellen, und dies umso mehr, als ein vergleichbares Dokument mit in solcher Weise kontinuierlich aufgezeichneten eigentlichen Kompositionen bisher ganz unbekannt ist.

Jede überprüfende Annäherung an diese Eintragungen muß zweifellos sehr sorgfältig, am besten in sauber begrenzten Schritten, erfolgen. Noch ohne daß dies bereits im Einzelnen erläutert wäre, lassen sie bald und leicht Gestaltungen und graphische Zeichen erkennen, von denen einige seltsamerweise auf Lautenmusik, andere auf Charakteristika der sogenannten Älteren deutschen Orgeltabulatur hinweisen. Vor allem im Blick auf die deutsche Lautenmusiknotation ist dies erstaunlich, da fortlaufende Proben von dieser erst aus Sebastian Virdungs Musica getutscht, ${ }^{9}$ gedruckt 1511, sowie - dabei aber mit vier eher pro memoria, als dem Lautenspiel dienen-

9 Vgl. Sebastian Virdung, Musica getutscht, Basel <, Michael Furter $>$ 1511, fol. M ii'$<\mathrm{M}$ iv $>$. 
den, intavolierten einstimmigen und rhythmisch nicht differenzierten Melodieniederschriften - in Berlin 719, dem sogenannten Königsteiner Liederbuch, ${ }^{10}$ mithin in der Zeit von etwa 1470-1473, bezeugt sind; Ältere deutsche Orgeltabulaturen dagegen sind schon vor 1460 belegt. Gegenüber der Lautentabulatur zunächst offenbart sich bald der grundsätzliche Unterschied, daß die in den Braunschweiger Fragmenten verwendete Notation eine Noten-, also eine Klang-, und nicht, wie die uns bisher vertraute deutsche Lautennotation, eine Griffschrift, mithin eine Tabulatur, ist; schon das schließt die Bruchstücke enger mit der Älteren deutschen Orgeltabulatur zusammen als mit jener eben erwähnten, erst späteren und prinzipiell abweichenden Art von Lautentabulatur, wie Virdung sie 1511 als Schöpfung des blinden Musikers Konrad Paumann bezeichnet. ${ }^{11}$ Die erwähnte Beziehung der Bruchstücke zur Lautenmusik, um das kurz und konkreter $\mathrm{zu}$ exemplifizieren, äußert sich in der Praxis, offensichtlich akkordische Klänge so zu notieren, daß zwei oder mehr, ja bis fünf übereinanderstehende „Notenköpfe“ an einer einzigen Cauda „hängen“ oder „hängen können"; auch daß die Notenköpfe bald oben, bald unten im System stehen, ohne daß die Koordination oder Separierung von oben oder unten verlaufenden „Stimmen“ im graphischen Verlaufsbild räumlich immer erkennbar und anschaulich würde, deutet auf Lautenmusik, die das in ihren später auftretenden deutschen Tabulaturen ebenfalls nicht immer darstellt und, vom Instrument her, einer spontan-improvisatorischen Spielweise überhaupt näher zu stehen scheint. Andere Zeichen gehören jedoch der aus Orgelintavolierungen des 15. und frühen 16. Jahrhunderts vertrauten Notation an: das gilt schon für die das Bild dort beherrschende mensurale Aufzeichnung von Musik, die sich wenigstens in den Ober-, nicht aber in jenen Mittel- und Unterstimmen findet, die eher „Griffe“ oder Instrumentaltasten, -höhen und -dauern mit Tonbuchstaben und rhythmischen Zusatzzeichen à la Virdung festhalten; in den uns vertrauten älteren deutschen Lautentabulaturen geschieht Ähnliches, indem sich diese bekanntlich eines, mit Buchstaben und Zahlen und davon getrennten, aber unanschaulichen Rhythmuszeichen arbeitenden Notationssystems bedienen. Der organistische Einfluß wird schließlich auch an der einen oder andern spezifischen Einzelheit deutlich, etwa der Kennzeichnung alterierter Tonhöhen durch ein vom Notenkopf abwärts führendes und schräg gekreuztes Strich-

10 Vgl. Paul Sappler, Das Königsteiner Liederbuch Ms. germ. qu. 719 Berlin, München 1970 (= Münchener Texte und Untersuchungen zur deutschen Literatur des Mittelalters ..., Bd. 29), S. 7; 323-328, zu Nr. 82; 375-380, zu Nr. 133-135, sowie Crawford Young und Martin Kirnbauer, Redaktion Thomas Drescher, Frühe Lautentabulaturen im Faksimile, Winterthur 2003 (= Pratica musicale, 6), S. 191-199.

11 Vgl. unten, S. 82ff. 
lein; ja selbst die tendentielle Praxis des Schreibers, Gruppen von befahnten kleinen Noten gleichen Wertes bei mehrheitlich ansteigendem Verlauf einzeln und mit Fahne, bei mehrheitlich absteigendem Verlauf dagegen ohne Fahnen, aber unter verbindendem Balken zu schreiben, findet sich hier - völlig übereinstimmend mit der Oberstimmenschreibung im Buxheimer Orgelbuch.

Glücklicherweise stehen diese Wolfenbütteler Fragmente aber doch nicht völlig allein. Im Jahre 1994 hat Christian Meyer aus dem Codex $2^{\circ}$ Ms. math. 31 der Landes- und Murhardschen Bibliothek Kassel eine in die zweite Hälfte oder das letzte Drittel des 15. Jahrhunderts zu datierende Zeichnung eines Lautenkragens publiziert und kommentiert ${ }^{12}$ (vgl. deren linke Hälfte auf Abb. 7e); ihr hat sich Martin Kirnbauer 2001 und 2003 erneut zugewandt und die dort erscheinenden Zeichen nochmals erläutert. ${ }^{13}$ Die Griffstellen an diesem Lautenkragen sind nicht mit den in der bisher bekannten deutschen Lautentabulatur üblichen Buchstaben und Zahlen, sondern mit den gebräuchlichen zusammengesetzten Solmisationssilben (nach dem Muster eeis/eelami/ffaut etc.), also mit Tonmarken, bezeichnet: das Kasseler Lautenkragen-Blatt ist seiner Funktion nach offenbar ein technischer Schlüssel, der helfen soll, musikalische Zeichen in der hier intendierten lautenmusikalischen Notation zu schreiben oder zu lesen - ein Werkzeug, wie es, im Prinzip übereinstimmend, auch aus dem 16. Jahrhundert bekannt ist. ${ }^{14}$ Der Verzicht auf die Angabe der Griffstellen ist, worauf hier bereits hingewiesen werden muß, vor allem deshalb bemerkenswert, weil die Virdung und bisher auch uns vertraute deutsche fünf-, dann sechs-chörige Lautentabulatur im Gegenteil mit Zahlen und Buchstaben, also mit der Angabe der Griffstellen, arbeitet; daß Virdung die Schöpfung dieser Tabulatur, wie schon erwähnt, dem blinden Musiker Konrad Paumann zuschreibt, ist keineswegs unwichtig und muß später nochmals erörtert werden.

Doch zurück zum Kasseler Blatt, das in eigenen kleinen Zusatzübersichten Mensural- bzw. Orgelnotenformen und -werte, aber nun besonders auch solche Notenzeichen ergänzt, die zwingend auf Lautenmusik hinweisen und weitgehend mit denjenigen der Braunschweiger Blätter überein-

12 Vgl. Christian Meyer, Eine Lauten-Unterweisung aus dem späten 15. Jahrhundert, Musik in Bayern, H. 49 (1994), S. 25-33.

13 Vgl. Martin Kirnbauer, „Possi stampar canto figurado ne intaboladure dorgano et de liuto “. Zur Problematik früher Instrumentaltabulaturen, Basler Jahrbuch für Historische Musikpraxis 25 (2001/02), S. 159-175, bes. S. 165-175; vgl. auch Anm. 10.

14 Vgl. z. B. Martin Staehelin, Neue Quellen zur mehrstimmigen Musik des 15. und 16. Jahrhunderts, in: Schweizer Beiträge zur Musikwissenschaft 3 (1978), S. 57-83, bes. S. 82f. 
stimmen. Die Zuordnung zum Lautenspiel geht dabei keineswegs nur aus der Zeichnung des Lautenkragens, sondern auch aus verschiedenen erklärenden Eintragungen, etwa aus einer Beischrift wie ,signa sursum traxionis“" (betrifft das Anreißen einer Lautensaite von unten), aus der Differenzierung ,in cantu“ bzw. ,in lutis“, hervor, mit denen in der Kasseler Zeichnung die übliche Noten- und die Lautengriffschrift von einander unterschieden werden; in der graphischen Darstellung von Semitonia stimmt die Kasseler Quelle mit den Fragmenten fast ganz, in derjenigen von Akkordklängen völlig überein.

Meyers kommentierender Text hat das Verdienst, als erster die in der Forschung langandauernde Vorstellung von damals je selbständigen Instrumentalwelten der Orgel und der Laute aufgebrochen und mit Nachdruck auf eine gewisse Nähe des Spiels von Orgel und Laute hingewiesen zu haben; später ist ihm Martin Kirnbauer aufgrund weitgehend derselben Quellendokumente gefolgt. ${ }^{15}$ Verständlicherweise lassen aber beide Autoren neben manchen schönen Quellenkombinationen, die sie beibringen, auch eine gewisse Unsicherheit erkennen, welche praktische Anwendung das auf dem Kasseler Blatt Aufgezeichnete wohl gefunden haben mag; Meyer beklagt am Schluß seines Beitrags ausdrücklich, es fehle nur „eine praktische Lautentabulatur in diesem System, welche die näheren Einzelheiten dieser eigenartigen Notationsweise zu präzisieren vermöchte “. ${ }^{16}$ Die hier vorzuführenden Braunschweiger Fragmente lösen dieses Desiderat ein.

3. Freilich, von einer eigentlichen Lautentabulatur kann, wie bereits betont, bei den neuen Fragmenten nicht die Rede sein, da sie keine Griffschrift belegen. Aber es liegt, um dieses Hauptergebnis gleich vorwegzunehmen, Lautenmusik vor, ${ }^{17}$ aufgezeichnet in einer Notation, die zwar Charakteristika dieser Musik widerspiegelt, sich aber verschiedentlich, ja vielfach an solche der älteren deutschen Orgeltabulatur anlehnt, dabei offenkundig bei durchgehaltener Meidung der dort üblichen Buchstabenzeichen für Mittelund Unterstimmen. Bestätigen läßt sich die Bindung dieser Musik an die Laute nicht nur mit den dem Kasseler Lautenkragen beigeschriebenen und übereinstimmenden Zeichen, sondern auch mit der besonderen Gestalt des Notentexts der Stücke (der übrigens in seiner gegenüber den vokalen

15 Vgl. Young/Kirnbauer (s. oben, Anm. 10), bes. S. 172-190. N.B. Die graphische Wiedergabe der Kasseler Zeichen auf S. 173 ist z.T. falsch, z.T. problematisch.

16 Meyer (s. oben, Anm.12), S. 33.

17 Der Aufsatz von Christopher Page, The 15th-century lute: new and neglected sources, Early Music 9 (1981), S. 11-21, bietet nur, wenngleich sehr willkommene, bildliche oder verbale Lautenzeugnisse des 15. Jahrhunderts, aber keine direkten Lautenmusik-Belege. 
„Konkordanz“-Fassungen durchweg etwas nach oben transponiert ist, wohl um den Tonumfang der Laute nicht zu überschreiten), schließlich auch damit, daß die Stücke sich nach Tonumfang und Akkordgriff-Möglichkeit auf einer fünfchörigen Laute gut spielen lassen. ${ }^{18}$

Dieses Ergebnis ist einigermaßen aufregend, weil die Musikforschung bisher überhaupt keine so frühe Lautenmusik-Aufzeichnungen kannte und damit von einer in gegebenen Kompositionen durchlaufenden Notation von Lautenmusik keine Kenntnis hatte. Bevor im Anschluß an diese Darlegungen die Braunschweiger Lautensätze, im Rahmen der Kleinüberlieferungs-Reihe hier also ausnahmsweise, in vollständiger Übertragung vorgeführt werden, erscheint es - um noch mögliche Zweifel an diesem Ergebnis zu zerstreuen und gleichzeitig dessen Folgen für Instrumentenbesetzung und Aufführungspraxis in den Blick zu bekommen - tunlich, nochmals kurz bei einigen weiteren Überlegungen zu verweilen.

Es könnte ja ein Zweifler zwar in den Fragmenten erhaltene Lautenmusik erkennen, aber eine solche, die einer nicht-lautenistischen Ausführung - nach den geforderten Akkorden dann wohl auf der zum mehrstimmigen Spiel fähigen Orgel - zugänglich gemacht werden sollte. Diese Auffassung ließe sich nicht nur mit den bei der Älteren deutschen Orgeltabulatur gemachten Zeichenentlehnungen der Braunschweiger Aufzeichnungen stïtzen, sondern auch mit der Vergleichbarkeit ihres Repertoires mit demjenigen, das in der gleichzeitigen süddeutschen Überlieferung der „Konkordanzen“" vorliegt, also etwa mit demjenigen in Berlin 40613 sowie in München 810, also demjenigen im Orgelteil des Lochamer Liederbuchs und im Buxheimer Orgelbuch. In diesem Zusammenhang muß besonders auf die Notiz „Jn Cytaris vel etiam Jn Organis“ hingewiesen werden, die eben das Buxheimer Orgelbuch, unter den Initialen Konrad Paumanns, der Orgelintavolierung eines weltlichen Satzes (Nr. 17) auf fol. 7, voranstellt. ${ }^{19}$ Die Harfe oder Harfen in der genannten Notiz hat David Fallows wegen der vom Satz geforderten Halbtöne - welche die Harfenstimmung damals kaum hätte wiedergeben können - ausgeschlossen, und Fallows und nach ihm auch Martin Kirnbauer haben als „Cithara“ hier mit Recht die Laute als Aufführungsinstrument vermutet. ${ }^{20}$ Dann freilich meint die Notiz offen-

18 Freundlich gewährte Auskunft von Herrn Kollegen Jürgen Heidrich (Münster/W.).

19 Vgl. München 3725, fol. 7-8, Nr. 17 (,Jeloymors M. C. C. b.“); Übertragung in Das Buxheimer Orgelbuch, Teil I ..., hrsg. von Bertha Antonia Wallner ..., Kassel etc. 1958 (= EdM 37), S. 14f.; dazu das (zum Teil falsche) Facsimile des Manuskripts, in: Documenta Musicologica, Kassel etc. 1955, fol. 7-8, Nr. 17.

20 David Fallows, 15th-century tablatures for plucked instruments: a summary, a revision and a suggestion, The Lute Society Journal 19 (1977), S. 7-33, bes. S. 31f., sowie Kirn- 
bar die wahlweise Instrumentenverwendung von Orgel oder Laute sogar zum Spiel aus der gleichen Musikalie; die beiden Autoren haben hier, mit Rücksicht auf den Tonumfang von zweieinhalb Oktaven, schließlich sogar an eine Besetzung mit zwei unterschiedlich großen Lauten gedacht, was immerhin bedenkenswert ist, weil beginnend vielleicht schon mit Spinacinos Tabulaturdrucken von 1507 angebliche Lautenduette belegt sind und Spielbeschreibungen dies auch andernorts nahelegen.

Außerhalb der oben berührten Eintragung im Buxheimer Orgelbuch sind keine direkten, ungefähr gleichzeitigen Zeugnisse doppelter Besetzungsmöglichkeit von erhaltenen Musikalien bekannt. Daß ein Organist um 1450/60 umgekehrt aus einer mit Buchstaben und Zahlen operierenden Lautentabulatur - hätte es sie denn überhaupt schon gegeben - gespielt hätte, ist unwahrscheinlich, denn er hätte dabei eine optisch leichter zugängliche Orgeltabulatur-Notation gegen eine optisch durchaus unanschauliche Tabulatur eingetauscht, damit erst noch eine solche, deren Information zur Griffstelle auf Saite und auf Bund auf den Orgeltasten vermutlich wenig brauchbar, ja vielmehr verwirrend gewesen wäre.

4. Es dürfte ratsam sein, nun einen genaueren Blick auf die Notentexte der Braunschweiger Fragmente zu werfen. Zunächst läßt sich feststellen, daß, den Textmarken entsprechend, hier weltliche, keine geistlichen Stücke aufgezeichnet sind. Wenn latinisiert-italienisch und deutsch bezeichnete Sätze direkt hintereinander stehen, fühlt man sich sogleich an ähnliche Werkfolgen erinnert, wie sie im Lochamer Lieder- und im Buxheimer Orgelbuch aus deutsch und italienisch oder auch französisch betitelten Stücken zustandekommen; auch die dichte Aneinanderschiebung der Eintragungen, wie sie einem etwa im Buxheimer Orgelbuch oft begegnet, kehrt hier wieder. Man wird sich in der Vorlagehandschrift der Braunschweiger Bruchstücke - wenn es eine solche überhaupt gegeben hat - ein ähnlich buntes Mischrepertoire versammelt zu denken haben, wie es die zwei oben genannten süddeutschen Vergleichsquellen aufweisen. Eine besonders enge Überlieferungsnähe zu diesen sollte man für die Fragmente jedoch trotzdem nicht postulieren: da uns aus dem mittleren 15. Jahrhundert vergleichbar umfangreiche norddeutsche Liedmanuskripte fehlen, wäre dieser Schluß

bauer (s. oben, Anm. 13), S. 169-171. - Den Plural „,Jn Cytaris“ sollte man, obwohl dies Fallows' Auffassung nicht widerlegt, nicht sogleich im Sinne einer Mehrzahl, hier also eines Duetts von Lauten, interpretieren, wie Fallows, S. 32, es tut, sondern bloß als Angleichung des Numerus an die direkt folgende Bestimmung ,Jn Organis“, die damals bedenkenlos auch für eine einzige Orgel gebraucht werden konnte. $\mathrm{Zu}$ den Lautenduetten vgl. auch Howard Mayer Brown, Instrumental Music before 1600. A Bibliography, Cambridge (Mass.) 1967, passim. 
vorschnell - denn wahrscheinlich gab es damals auch im nördlichen Deutschland ein gewisses, aber heute weitgehend verlorenes Repertoire ein- und mehrstimmiger weltlicher Lieder und Liedsätze, in das sich die Braunschweiger Bruchstücke natürlich und nahe eingeordnet hätten. ${ }^{21}$

Die Übersicht über die Aufzeichnungen selber bestätigt, daß man in ihnen tatsächlich fast alle Zeichen wieder finden kann, welche dem Kasseler Lautenkragen beigeben sind; selbst die Notenköpfe sind, wie dort, nicht in geschlossen körperhafter Form, sondern mit einem der cauda beigebenen senkrechten Parallelstrichlein bezeichnet, das meist nicht einmal mit der zugehörigen cauda verbunden ist, und die Übereinstimmung mit der Kasseler Zeichnung geht sogar bis zum Wiederholungszeichen, das nicht zwei senkrechte, sondern zwei gespiegelt konvexe Halbrundstriche mit Schlangenlinien zusammenbindet, seitlich je durch zwei senkrechte Doppelpunkte flankiert. Von der Nennung noch weiterer Entsprechungen sei hier abgesehen; im Bedarfsfall sind sie in den genannten Publikationen von Christian Meyer und Martin Kirnbauer samt Erläuterungen leicht $\mathrm{zu}$ finden. Es ist aber lohnend, hier noch einige andere Beobachtungen an den Quellen anzufügen.

Bemerkenswert erscheint, daß die Notensysteme in den Braunschweiger Bruchstücken, wie schon erwähnt, durchweg gleichermaßen aus acht Linien bestehen, so unkontrolliert in ihrer Länge und in der Einhaltung gleich großer Binnen-Zwischenräume jene oft ausgefallen sind. Überhaupt scheint hier so etwas wie ein bereits übliches und selbstverständliches Darstellungsmuster unangefochten eingehalten worden zu sein. Dies mag sich schon in den fallenden Tonhöhenvorzeichnungen am linken Rand der Systeme andeuten, die, wo sie erhalten sind, mithin fast durchgehend, mit gg, cc, f, c und g markiert sind: es läßt sich keine Tonhöhenvorzeichnung und -position finden, die je nach dem besonderen Bedarf des gerade aktuellen Stückes gewählt worden wäre, und man bekommt hier vielmehr den Eindruck, daß die Eintragungen auf der Grundlage einer überkommenen

21 Parallele Recherchen nach sicher datierten bildlichen Lautendarstellungen in Norddeutschland sind wenig erfolgreich verlaufen. Vor einen Lautenengel auf der geschnitzten Türe eines Sakristeischranks mit dem Datum 1425 im Hamburger Museum für Kunst und Gewerbe (Inv. Nr. 1895.189) war zeitlich nicht zurückzukommen. Dieser Gesamtbefund könnte jedoch die langsamere Akzeptanz des Instruments in Norddeutschland bestätigen, die das Vordringen der Laute aus Arabien über Spanien vor allem nach Italien und Frankreich, viel weniger aber über den Süden Deutschlands hinaus in dessen Norden begünstigt zu haben scheint; vgl. dazu auch Karl Geiringer, Vorgeschichte und Geschichte der europäischen Laute bis zum Beginn der Neuzeit. Eine ikonographische Studie, ZfMw 10 (1927/28), S. 560603, bes. S. 574. - Frau Dr. Christine Kitzlinger, Hamburg, sei für freundliche Auskünfte bestens gedankt. 
und allgemein gültigen Praxis notiert worden seien. Und damit nicht genug: auch der Tonumfang des Instruments wird, ohne eine Überschreitung nach oben oder unten in allen Stücken von oben g" an fallend bis g eingehalten: die Eintragungen richten sich durchaus nach den Möglichkeiten jenes fünfchörigen Instruments, das offenbar benutzt wurde, mithin mit einem Umfang von zwei Oktaven. Dieser ist etwas kleinräumiger als derjenige, den das Buxheimer Orgelbuch in der oben erwähnten ,Jn cytharis vel Jn organis"-Eintragung mit Rücksicht auf die dort möglicherweise zwei spielenden Lauten voraussetzt. Übrigens sind die zwei Oktaven in den Fragmentniederschriften fast so umfangreich, wie der Tonraum, den die Laute Virdungs für ihren 2. bis 5. Chor zur Verfügung stellt. Man darf auch von allem diesem her annehmen, daß hier Lautenmusik vorliege. Übrigens scheint es auch, daß diese Braunschweiger Sätze auf nur einer Laute gespielt wurden; für zwei Lautenisten wäre das Bild der Eintragungen wohl nicht rasch genug und hinreichend übersichtlich, zumal auch nicht zwei deutlich getrennte Eintragungsfelder wie im Buxheimer Orgelbuch vorgesehen waren und die Oberstimme mitunter einiges kleinwertiges Rankenwerk in ihren Vortrag einbauen konnte und wohl auch sollte.

Das graphische Bild der Eintragungen macht zunächst einen etwas ungeschickten Eindruck, allerdings können auch die Federproben und sonst zugefügten Schnörkel, auch die Verschmutzung und Beschädigung des Papiers, diesen Eindruck verstärken. Man sollte diesem aber nicht allzu sehr nachgeben, denn die hier tätige Kopistenhand schreibt - wie sich zeigt, wenn man sich etwas eingelesen hat und einmal die ohnehin problematische Akzidentiensetzung übergeht - dann doch in ihrer Weise sicher, zielgerichtet und vermutlich sogar einigermaßen fehler- und korrekturarm: man wird das, was das äußere Bild der Handschrift leicht ungeschickt wirken läßt, deshalb eher als Ausdruck der individuellen Persönlichkeit des Kopisten ansehen, eines Kopisten, der etwas vom Lautenspiel verstand und der, da er wohl auch der direkt aus diesem Gebrauchsmanuskript spielende Lautenist war, ebenfalls für die besondere Gestalt der Stücke verantwortlich war. Diesen Eindruck werden die Übertragungen nochmals vermehren.

Der Prozeß und die Darbietung dieser Übertragungen haben, wie hier deutlich festgehalten werden muß, mit einigen erheblichen, teilweise miteinander engverbundenen Grundsatzschwierigkeiten zu kämpfen. Einmal disponieren die Niederschriften ihren Verlauf, wie erwähnt, ohne alle taktartigen Striche oder Zwischenräume, also nicht so, daß die rhythmischen Werte der Noten sich sogleich zu einer gegliederten und erkennbaren sowie verständlich ,gekammerten“ Gesamtstruktur zusammenfügen würden. Das gilt umso weniger, als hoch und tief gelegene Einzelnoten nicht selten 
in mutmaßliche „Klangräume“ gegensätzlicher Höhen- oder Tiefenlage fallen, die, dem Manuskript gemäß, dann gerade nicht anklingen, sondern zur gleichen Zeit vielleicht nur „ausgehalten“ werden, allerdings ohne daß dieses gegenseitige Verhältnis graphisch deutlich festgehalten worden wäre. Die gesamten horizontalen Zusammenhänge der Noten innerhalb eines Melodieverlaufs - sollen sie ineinander erklingen oder sich ablösen? - sind danach begreiflicherweise ziemlich schwer zu durchschauen, was auch rhythmische Folgen haben muß. Dazu gesellt sich die zweite Schwierigkeit, daß die hier aufgezeichneten Sätze zwar mehr oder weniger klar vokale oder orgelgebundene Vorlagemelodien verarbeiten, wie sie in den „Konkordanz"- oder Vergleichsquellen ${ }^{22}$ erhalten geblieben sind; die Bemühung, ein präzises Konkordanzverhältnis festzustellen, muß freilich, wegen der damals möglichen Nähe der Gesangspraxis auch zum nicht-schriftlich tradierten einstimmigen Liedgesang, damit rechnen, daß einerseits bereits die Vorlageweisen modifiziert, ja gar in ,zersungener“ Gestalt den Weg in die erhaltene Manuskriptüberlieferung gefunden haben, andererseits ein gleicher Prozeß auch den Lautenniederschriften vorausgegangen ist. Da diese, anders als die vokalen oder Orgelquellen, keinen deutlich stimmigen Satz bieten und sich mitunter wohl auch einer spezifisch lautenistischen Improvisationsfreude „cum admirandis modulorum superinventionibus" in der ,suprema pars cujusvis compositi cantus“, wie Johannes Tinctoris sagt, ${ }^{23}$ öffnen oder doch öffnen können, erbringt der Vergleich mit den vokalen oder organistischen Konkordanzfassungen bisweilen keineswegs rasche und klare Ergebnisse darüber, ob eine Konkordanz vorliege und was davon in den Lautensatz übergegangen sei. Und da, wenngleich in einer frühen italienischen, in Bologna liegenden Lautenquelle, ein dreistimmiger Chansonsatz von Vincenet mit in einem eigenen Notensystem in Mensuralnotation geschriebener Oberstimme und den zwei in italienischer Lautentabulatur

22 Bei instrumentalen Bearbeitungen von Vokalsätzen oder -melodien jener Zeit ist der strenge Begriff der Konkordanz als einer grundsätzlich gleichen Parallelfassung bekanntlich problematisch, weil die sogenannte Konkordanzenlage dann nicht selten auch in sich vielfältig, ja wirr und unübersichtlich ist. Deshalb beschränken sich die oben gebotenen Angaben gewissermaßen auf große und reichhaltige Vergleichsquellen, und dies umso mehr, als diese zum Vergleich in Facsimile- oder Gesamtausgaben leicht zugänglich sind und darüber hinaus das ausgezeichnete Buch von David Fallows, A Catalogue of Polyphonic Songs, 1415-1480, Oxford 1999, alle erwünschten und nötigen Konkordanzangaben für sämtliche in den Braunschweiger Fragmenten vertretenen Sätze bzw. Textmarken, also auch etwa zur einstimmigen Liedüberlieferung u.v.a., gewährt.

23 Vgl. Karl Weinmann, Johannes Tinctoris (1445-1511) und sein unbekannter Traktat „De inventione et usu musicae“, Regensburg 1917, Repr. Tutzing 1961 (dem hier gefolgt wird), S. 45. 
aufgezeichneten Unterstimmen erhalten ist, ${ }^{24}$ ergibt sich als eine dritte Schwierigkeit die Frage, ob in den Braunschweiger Fragmenten mitunter vielleicht auch eine Art reduzierten Begleitsatzes zu einer vokal aufzuführenden Oberstimme vorliegen könnte, wodurch die musikalische Anlehnung des notierten Lautensatzes an eine Vorlagemelodie nochmals schwerer wahrnehmbar würde. Da diese Besetzung in späteren Lautenquellen tatsächlich vorgesehen ist, ${ }^{25}$ sollte diese Möglichkeit, auch wenn sie hier nicht wirklich zuzutreffen scheint, von Lautenhistorikern, die sich mit den vorliegenden Fragmenten beschäftigen werden, mitbedacht werden; hier wird von einer weiteren Abklärung abgesehen, damit der quellenkundliche Charakter des Textes nicht überdehnt wird.

Die Folge aller dieser Beobachtungen und Überlegungen kann nur sein, daß die Umschriften im Folgenden möglichst wenig „Interpretation“ erfahren sollen. Dem Leser wird also nur eine möglichst rasche Lesehilfe für das angeboten, was überliefert ist. Deshalb sind die Sätze so wie in der Quelle notiert, also auch ohne Transposition, übertragen, ebenfalls ohne Ergänzung eines Gerüstes von Taktstrichen oder anderer, Raum und Verlauf ordnender Hilfen (obwohl ein solches für die rasche Erkenntnis einer Vorlagenverarbeitung mitunter hilfreich sein könnte); nur Doppelstriche und Wiederholungszeichen werden, wo die Konkordanzen-Überlieferung dies aufzeigt, eingefügt. Auch werden Noten nicht verlängert, in die später einsetzende hineinklingen - der Leser soll möglichst unvoreingenommen weiterdenken und -schließen können; die hier beigegebenen Abbildungen zeigen überdies das originale Quellenbild.

[Die Übertragungen der Sätze folgen am Schluß dieses Abschnitts, auf S. 85-88.]

Die Übertragungen und die in ihnen festgehaltene Musik sollen hier nur kursorisch besprochen werden, denn bis in deren differenzierte Analysen sollte eine spezifisch quellenkundliche Arbeit wie die vorliegende nicht ausgedehnt werden; der Leser sei freundlich aufgefordert, die Vergleichsrecherchen selbständig zu unternehmen. Hier empfehlen sich aber wenigs-

24 Bologna, Biblioteca Universitaria, Ms. 596. HH. $2^{4}$, fol. $1^{\prime}-2$ (= neu 3'und 1). Vgl. dazu Fallows (s. oben, Anm. 20), S. 18-28 mit Plate 4-5.

25 Z. B. Francesco Bossinensis, Tenori e contrabassi intabulati col sopran in canto figurato per cantar e sonar col lauto, libr. I und II, Venedig, Ottaviano Petrucci, 1509, Nr. 170, und 1511, Nr. 1-56, oder Paris, Bibliothèque Nationale, Vmd. Ms. 27. Im deutschsprachigen Gebiet ist diese Besetzungspraxis in gedruckten Zeugnissen erstmals belegt bei Arnolt Schlick, Tabulaturen Etlicher lob gesang und lidlein ..., Mainz 1512, Nr. 15-26. 
tens noch einige Worte zum jeweiligen Verhältnis von Lautensatz und Vorlagenverarbeitung.

Die Textmarke Cum lacrimis, um auch hier der vorher eingehaltenen Reihung der Sätze zu folgen, ruft sogleich die seinerzeit beliebte zweistimmige Ballade Con lagreme bagnandome von Johannes Ciconia ${ }^{26}$ in Erinnerung. Allerdings macht es große Mühe, in dem entsprechenden Lautensatz mit Sicherheit eine Bearbeitung von Ciconias Stück zu erkennen, und dies weniger, weil jener in dem Fragment durch Beschnitt und Beschädigung einen wesentlichen Textverlust hat erleiden müssen, als vielmehr, weil, wenn wirklich Ciconias Ballade die Vorlage gewesen wäre, die Übereinstimmung der beiden Vergleichsfassungen geradezu bedenklich beschränkt geblieben wäre. Gewiß gibt es einige kurze Tonfolgen, die vergleichbar sind, und man möchte auch an einigen Stellen überhaupt formal „Ciconia-Typisches“ vermuten, aber die Balladenvorlage scheint insgesamt so schwach durch, wie dies in den mehreren Orgel-Arrangements im Lochamer Lieder- und im Buxheimer Orgelbuch niemals der Fall ist. Man wird sich deshalb fragen wollen, ob die fragliche Textmarke nicht etwa irrtümlich über den Satz in dem Braunschweiger Fragment geraten ist und in Wirklichkeit ein anderer, aber bisher nicht wiedererkannter Satz Ciconias oder auch eines anderen Verfassers Vorlage gewesen ist.

Myn trud gheselle läßt zunächst schwanken, ob hier der, angeblich auf den Mönch von Salzburg zurückgehende T oder der D jenes dreistimmigen Satzes verarbeitet ist, der in den beiden Vergleichsquellen vorliegt. Prüft man den Lautensatz sorgfältig vor allem in seinem Stollen, so scheint die Übereinstimmung mit dem D eher größer. Leider ist von der Fortsetzung, die hier vielleicht aussagekräftig gewesen wäre, nur noch etwa die Hälfte überliefert; der Rest ist verloren.

Gruß senen jch im hertzen traghe folgt im oberen Bereich des Lautensatzes ziemlich übereinstimmend dem D des dreistimmigen „Konkordanz“-Satzes im Schedel-Liederbuch.

In Jch fare do hyne wen e $\beta$ mu $\beta$ syn zeigt sich die Vorlagemelodie, wenn man ihre einzige und einstimmige Vergleichs-Fassung heranzieht, nicht durchgehalten in der oberen Region des Lautenarrangements, sondern mehrfach in dessen Mittellage. Man könnte sich fragen, ob dem Lautenisten damals eine melodisch zum Teil zersungene oder auch „übersungene“ vokale Fassung bekannt war.

26 Der Satz ist ediert in Polyphonic Music of the Fourteenth Century, vol. XXIV (= The Works of Johannes Ciconia, ed. Margaret Bent / Anne Hallmark), Monaco 1985, S. 130f., Nr. 29., sowie S. 210f., Nr. 29. 
Ellende du hest vmb vanghen mich: Der hier anscheinend nur zu etwa zwei Dritteln überlieferte Satz legt die in der einstimmigen VergleichsÜberlieferung in Berlin 40613 gebotene Liedmelodie, ähnlich wie eben vorher in Jch fare do hyne..., in die Mittellage der Lautenfassung; man muß sie sich dort, am besten im direkten Vergleich mit der genannten Liedmelodie, gleichsam in Einzeltönen zusammensuchen.

5. Der Braunschweiger Fund fordert schließlich beinahe gebieterisch, in die Geschichte des frühen Lautenspiels in Deutschland, seiner Musik und seiner Aufzeichnungsart wenigstens in knappen Zügen eingeordnet zu werden. So aufregend der Fund auch sein mag, so entschieden muß man jedoch betonen, daß das derzeitige Gesamtbild dieser frühen deutschen Lautengeschichte noch immer an vielen Stellen voller unbeantworteter, ja unbeantwortbarer Fragen und damit eine in Einzelheiten klare Übersicht dementsprechend ungewiß ist und, wenn ein solche überhaupt versucht werden soll, diese dann nicht frei von spekulativen Zügen sein kann. Trotzdem sei sie riskiert, und dies schon deshalb, weil so die Lautenhistoriker dazu angeregt werden, sich mit den hier vorgeführten Sachverhalten und Erwägungen produktiv auseinanderzusetzen.

Johannes Tinctoris kommentiert in seinem in den Jahren nach 1480 verfaßten Traktat De inventione et usu musicae Spielsituation und -eigenheiten der Laute (die er als „lyra“" und, gemäß populärem Wortgebrauch, als „leutum“ bezeichnet): „Usus autem ipsius lyrae quam leutum vulgo nuncupari prediximus: festis, choreis et conviviis privatisque recreationibus apud nos inservit. In qua [besser: quo? oder: quibus?] plurimi precipue germani eximie sunt eruditi“. ${ }^{27}$

Es ist auffällig, daß Tinctoris hier den Deutschen einen besonderen Sinn für und eine engagierte Beteiligung an festlichen Lautenspielgelegenheiten zuschreibt; man fragt sich sogleich, ob der in den Braunschweiger Fragmenten erhaltene Repertoire-Ausschnitt gerade mit weltlichen Sätzen diese Auffassung Tinctoris' bestätigen könnte. Aber noch wichtiger erscheint, daß Tinctoris nicht nur hier den Deutschen überhaupt mehrfache Verdienste um das Lautenspiel zuerkennt; das gilt auch an anderer Stelle, an der er den Ausbau des Instruments durch die allmähliche Vergrößerung der Saiten- und Chorzahl geradezu entwicklungshistorisch beschreibt: Im Altertum „lyra septem chordas inter se tonis ac semitonis differentes detendisse fertur ... Sed quom ipse chorde, per tonos ac semitonia discrepantes, non omni composito cantui suppetebant, quinque et aliquando sex principalium ordinatio ea subtilitate a posteris (ut reor) Germanis inventa est, ut ... lyra

27 Vgl. Tinctoris (s. oben, Anm. 23), S. 45. 
sit perfectissima. Quin ut fortiorem habeat sonum, cuilibet istarum chordarum una conjungitur ... His autem chordis ex arietum intestinis communiter factis sunt qui germanica inventione gravissime quandam aliam ei ... consonantem adjiciunt eneam, qua concentus non modo itidem fortior, verum etiam longe suavior efficitur" ${ }^{28}$ Zuerst also besa $\beta$ das Instrument sieben Saiten, aber das reichte nicht für jedes Ensemblespiel, so daß nach einer Erfindung der später folgenden Deutschen - und jetzt springt Tinctoris von der Saiten- zur Chorzahl - fünf und gelegentlich sechs Chöre angebracht wurden. Und wiederum nach einer deutschen Erfindung wurde dann nochmals ein neuer tiefster Chor ergänzt, ein Vorgang, wie er sich als nachträglicher Schritt durch die vom sonstigen System der deutschen Lautentabulatur abweichende Griffstellen-Bezeichnung deutlich zu erkennen gibt.

Vergleicht man diese zweite, in der Lautenliteratur bisher offenbar nicht herangezogene Tinctoris-Äußerung mit der oben bereits berührten Virdung-Stelle, so erkennt man leicht eine prinzipiell enge Übereinstimmung. Auch jenes Virdung-Zitat sei hier verkürzt wiedergegeben: ,Jch höre das ayn blind, zů nürenberg geborn vnd zů münchen begraben, sie [sei] gewesen, hatt meister Conrat von nürenberg gehaissen, der zů seyner zeytt vor ander instrumentisten gelopt vnnd gerümptt sey worden. Der hatt auf den kragen der Fünff köre vnnd vff siben bünde das gantz alphabet haissen schreiben ... Aber hernach sind ettlich andere künnen [= kommen?], der ich eyns tails die ersten anfenger von hören sagen gesehen hab, die eben auch die selb tabulatur also gebraucht, wie er sye für gegeben hatt, unnd noch zwo saitten, das ist den sexten kor, dazů gethon ....". ${ }^{29}$ Die Übereinstimmung ist deutlich: Der aus humanistischer Gesinnung am antiken Ideal des „primus inventor“ einer Sache bestimmt sehr interessierte Tinctoris kann zwar Conrad Paumann nicht namentlich nennen, aber seine unbenannten „Germani“ entsprechen doch unverkennbar den ebenfalls namenlosen „ettlich anderen“, die in Deutschland angeblich auf Paumanns Leistung aufgebaut haben und die Virdung noch gesehen haben will. ${ }^{30}$ Versucht man, die bereits oben benannte Schöpfung Paumanns in dieses ganze Spektrum der deutschen Lauten-Innovationen einzuordnen, so wird dies

28 Vgl. Tinctoris (s. oben, Anm. 23), S. 41.

29 Vgl. Virdung (s. oben, Anm. 9), fol. K iij'.

30 Zur Diskussion dieser Aussage Virdungs vgl. Franz Krautwurst, Bemerkungen zu Sebastian Virdungs „Musica getutscht“ (1511), Festschrift Bruno Stäblein zum 70. Geburtstag, hrsg. von Martin Ruhnke, Kassel etc. 1967, S. 143-156, bes. S. 148 153. Im Blick auf die folgenden Schlußgedanken sei festgehalten, daß die Ausführungen von Crawford Young in: Young/Kirnbauer (s. oben, Anm. 10), S. 10ff., zum Verhältnis von früher lautenistischer Schriftlosigkeit und folgendem Aufkommen schriftlicher Lautennotation sehr bedenkenswert erscheinen. 
deshalb leicht möglich, weil die entsprechende, von Virdung dem blinden Paumann zugeordnete Tabulatur-Leistung von ihrer Eigenart her erhebliche Glaubwürdigkeit beanspruchen kann: Denn die angeblich Paumannsche Erfindung paßt deshalb genau zu einem Blinden, weil sie jeden Lautenton-Griff mit einem einzigen Tabulaturzeichen vollständig markiert und damit, anders als alle anderen Lautentabulatur-Schriften der bald folgenden Zeit, auf die für einen Blinden entschieden kompliziertere Griffbezeichnung mithilfe zweier Merkmale, mithin sowohl der jeweiligen Saite als auch des jeweiligen dort geforderten Bund-Griffs, verzichten kann.

Die angeblich Paumannsche Tabulatur-Neuerung scheint damit also eine individuelle Erfindung ihres Urhebers gewesen $\mathrm{zu}$ sein, nicht eine in langer sukzessiver Entwicklung allmählich herangewachsene TabulaturEndform; schon in den frühen siebziger Jahren des 15. Jahrhunderts ist sie, wie die knappen Eintragungen im Königsteiner Liederbuch zeigen, verwendet worden..$^{31}$ Mit dem individuellen und ereignishaften Charakter der Paumannschen Tabulaturpraxis ist aber zugleich der Schluß geradezu gefordert, daß es vor dieser Umstellung eine andere Schriftpraxis für Lautenmusik gegeben haben muss.

Diese Einsicht schwächt jeden Gedanken etwa der Art, daß in den Braunschweiger Fragmenten eine bloß individuell-experimentelle und einzelfall-bedingte Umschrift vorgelegen haben könnte, die mit ähnlich didaktischer Absicht wie die auf der Kasseler Lautenkragen-Zeichnung enthaltenen Informationen weltliche Stücke, die in einem mittlerweile verlorenen Lautentabulatur-Manuskript à la Paumann notiert gewesen wären, unter Heranziehung von Elementen der älteren deutschen Orgeltabulatur einem jener Lautentabulatur unkundigen Organisten hätte spielbar machen sollen. Denn zum einen wäre ein solcher Spezialfall eben deshalb nicht belegbar und somit wenig beweiskräftig; zum andern wirken die Braunschweiger Bruchstücke als Zeugen einer ganz der musikalischen Aufführungspraxis zugedachten Handschrift; und zum dritten sind die Ursprungsregionen der hier herangezogenen Dokumente, mithin Nordhessen und die Braunschweiger Gegend, geographisch so weit voneinander separiert, daß man

31 Es scheint erwähnenswert, daß die förmliche „Erfindung“ der deutschen Lautentabulatur nicht nur von Tinctoris und Virdung (s. oben), sondern auch von Hans Judenkünig, Ain schone kunstliche vnderweisung in disem büechlein / leychtlich zu begreyffen den rechten grund zu lernen auff der Lautten vnd Geygen ..., <Wien> 1523, Beginn der Vorrede, festgehalten worden ist: „Es ist menigelich wissen / das in kürtzen jaren bey man $\beta[=$ Menschen] gedechtü $\beta$ / erfunden worden ist die Tabalatur / auff die Lautten ....". Man mag überdies fragen, ob die erwähnten deutschen Bemühungen um Lauteneinrichtung und -ausstattung die deutliche Präsenz deutscher Lautenmacher in Italien (Laux Maler in Bologna u.a.) erklären können. 
sie nicht aus einem ex eventu konstruierten Einzelfall in eine enge Verbindung bringen dürfte. Die Gegenden ihrer Entstehung fordern vielmehr die Annahme, daß es vor der Paumannschen Tabulaturform eine ältere, damals ebenfalls verbreitete, aber bis heute wegen vielfältiger Quellenverluste kaum faßbare Schriftpraxis von Lautenmusik gegeben haben muß. Nach den oben vorgeführten, an voneinander entfernten Orten Deutschlands entstandenen Zeugnissen aus Hessen und der Braunschweiger Gegend und nach der in den hier vorgestellten Fragmenten beobachteten Stabilität der schriftlichen Darbietung ist man geradezu gezwungen, die hier geübte Niederschrift von Lautenmusik eben für ein Zeugnis jener älteren Schriftpraxis zu halten: die Datierungen der Quellen passen ohne weiteres in dieses Gesamtbild und erklären gleichzeitig die seltene Erhaltung solcher älterer schriftlicher Lautenmusik-Quellen. Natürlich wird man sich schließlich fragen, warum die ältere Praxis zur Niederschrift die entschiedene Orientierung gerade an der älteren deutschen Orgeltabulatur gesucht habe. Die Antwort scheint naheliegend zu sein: weil die Orgeltabulaturschrift, wie sich ohne Mühe an erhaltenem Material belegen läßt, bereits bestand und im deutschen Gebiet offenbar verbreitet war, die Lautenmusik jedoch bis ins 15. Jahrhundert hinein wohl noch weitgehend frei improvisiertem Spiel unterlag und erst aus einem zeitgemäß neuen Bedürfnis nach Verschriftlichung die Hilfe bei der Orgeltabulatur suchte und fand. Wenigstens scheint sich so eine ziemlich glaubwürdige Folie für einen Vorgang zu ergeben, der leider nur in wenigen Dokumenten bezeugt ist, aber sich in seinen Teilen auf diese Weise plausibel zusammenzufügen scheint. Die Braunschweiger Fragmente bilden einen unüberschätzbaren Beitrag zur Erkenntnis dieses Vorgangs. 
7. Norddeutsche Fragmente mit Lautenmusik

85
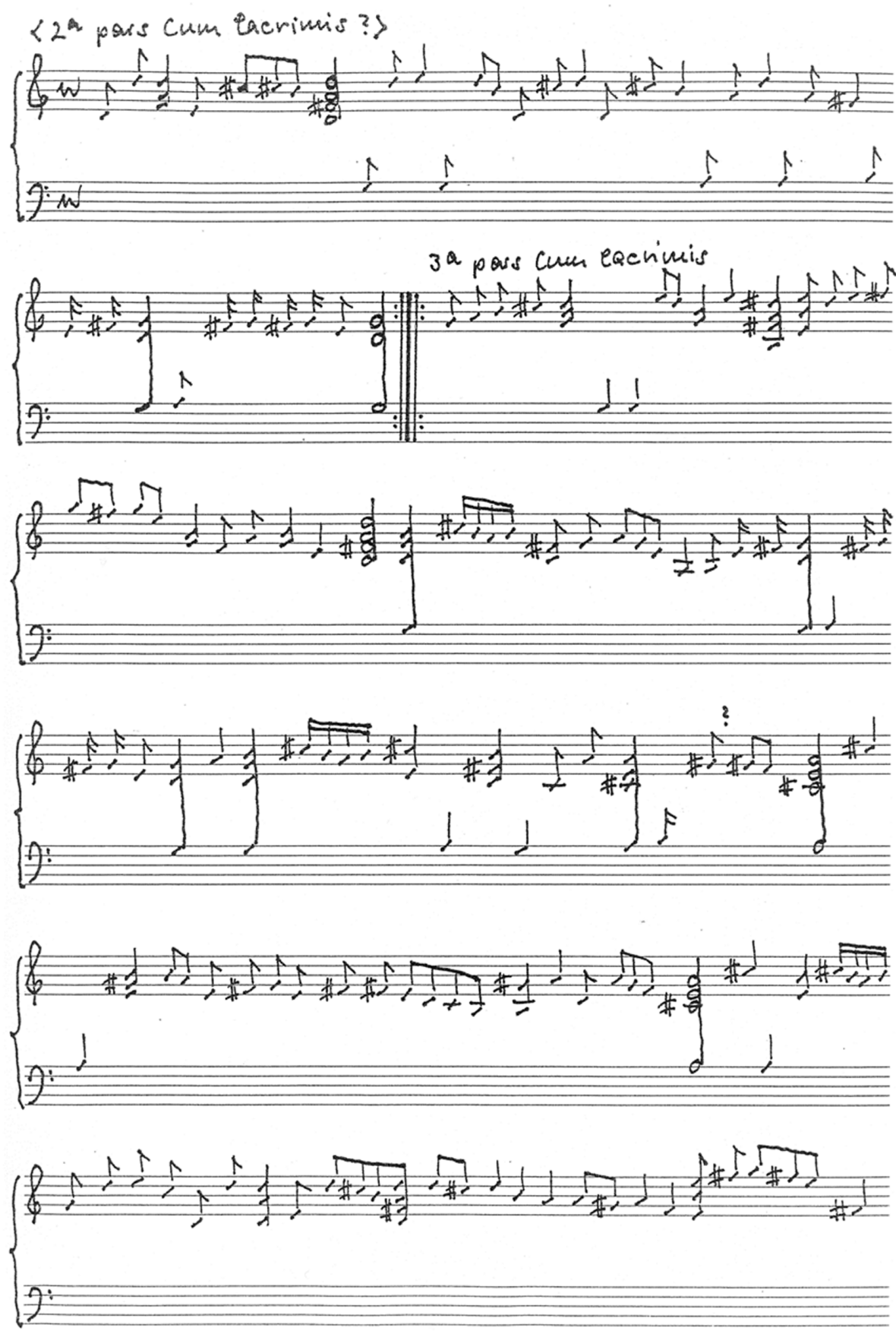
86

Martin Staehelin
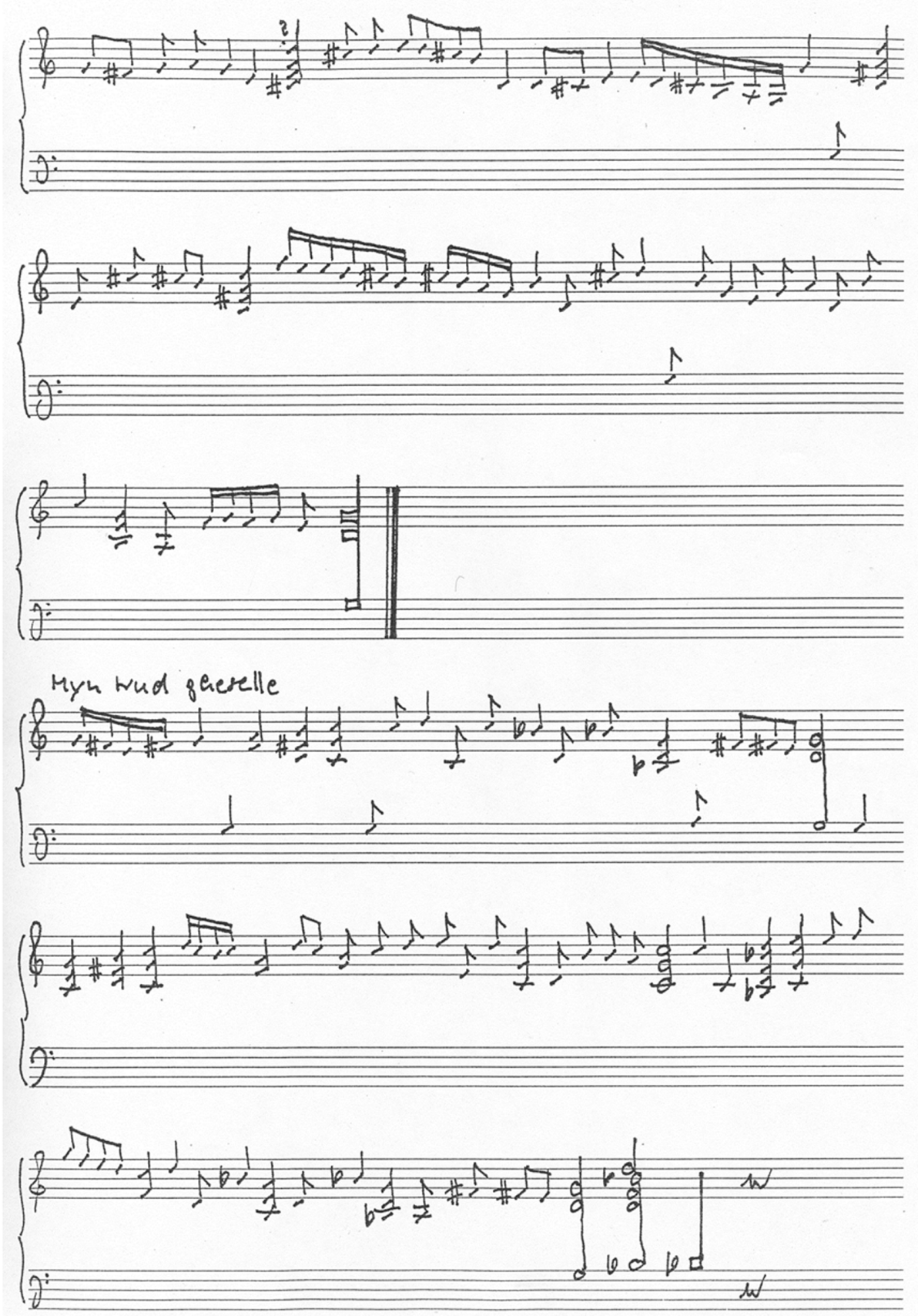
Grup senen ich im hertzen traghe
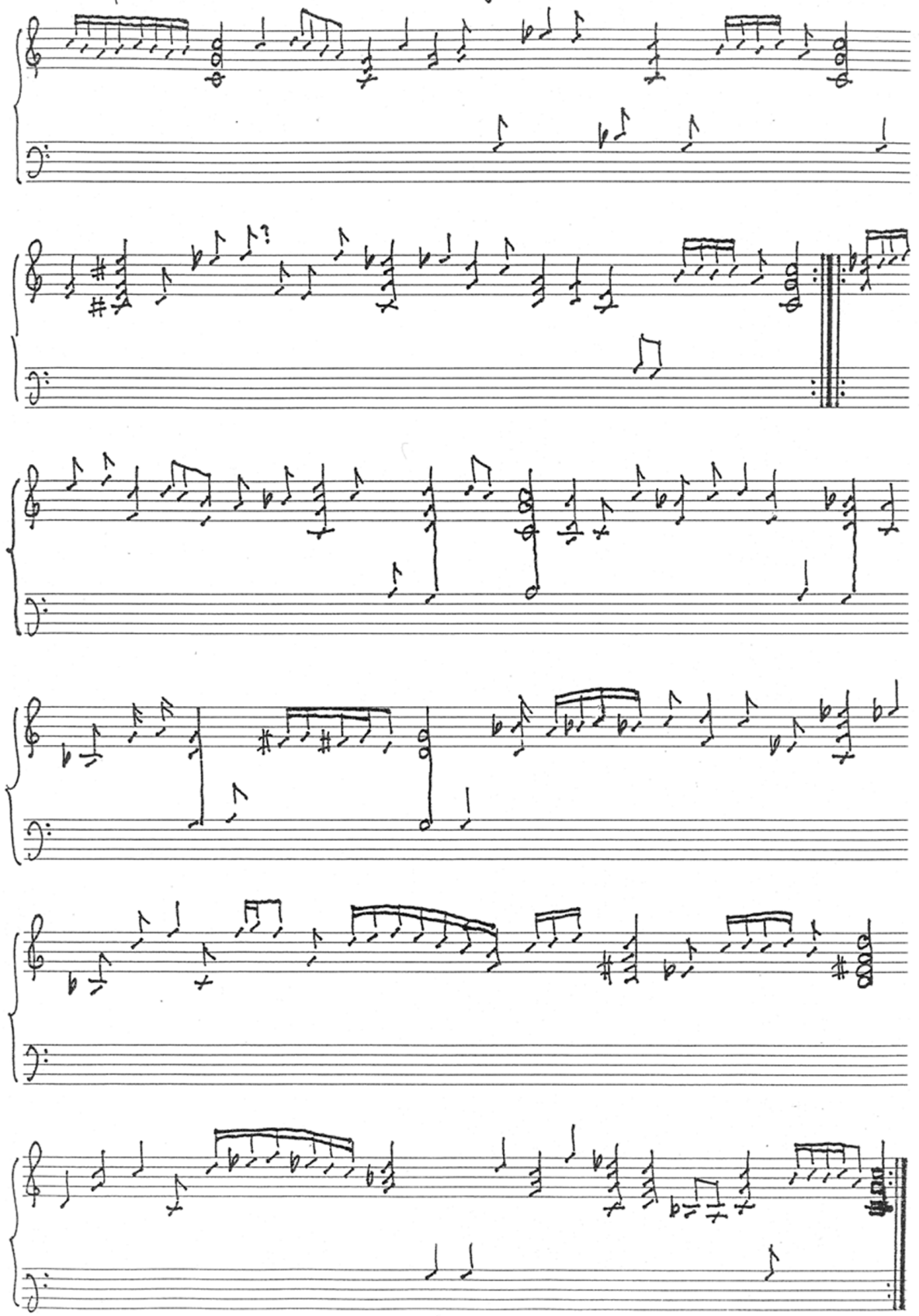
Jeh fare dohyne ben es was dyn

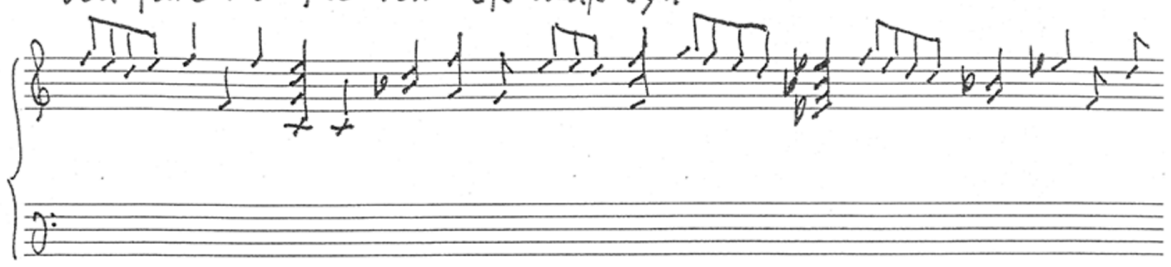

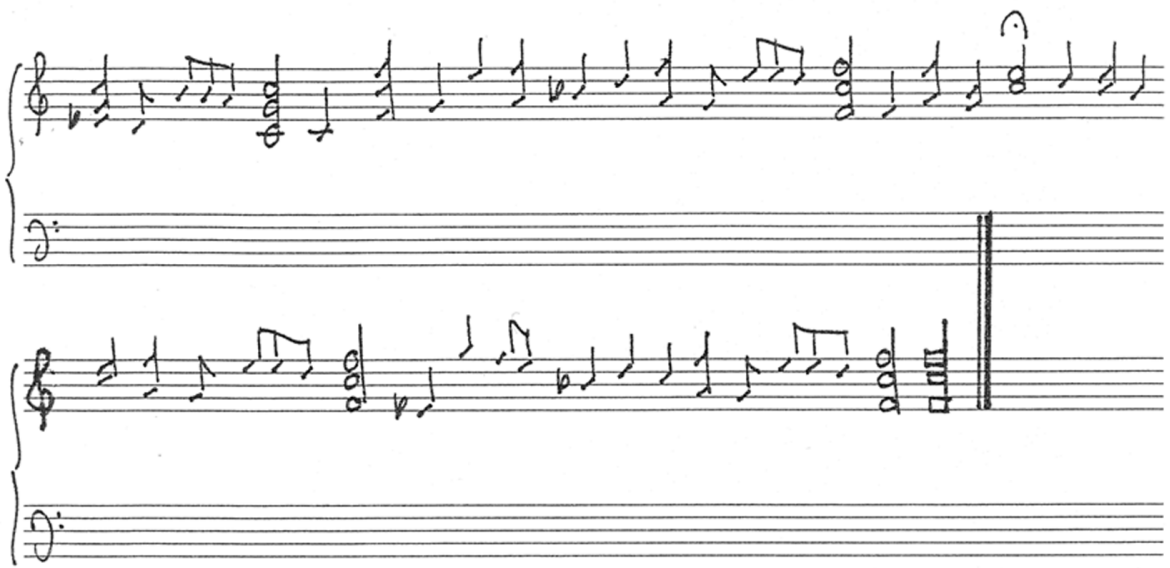

Ellende du hest rmb vanghen wich

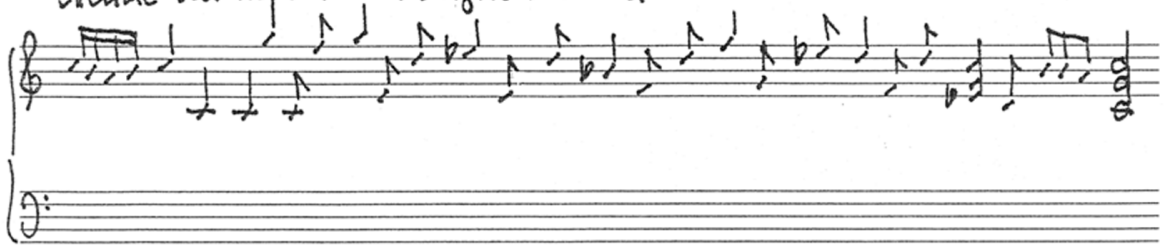

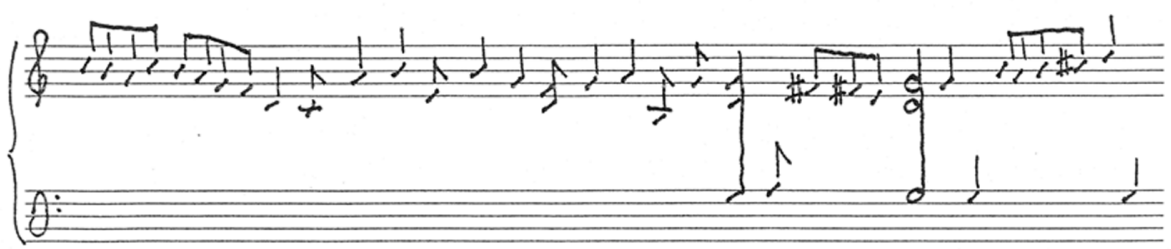

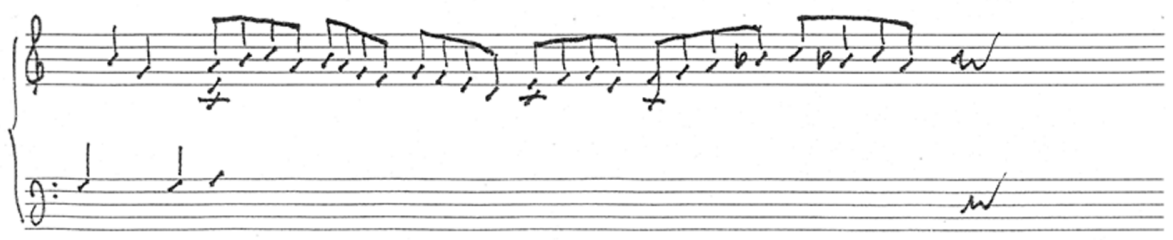

